\title{
High Intake of Maternal Milk Prevents the Development of Mammary Cancer in Pups Maintaining Elevated Ingestion of Saturated Fat
}

\author{
Flavia Eliana Santiano ${ }^{1,2}$, Corina Verónica Sasso ${ }^{1}$, Leila Ester Zyla ${ }^{1}$, Flavia Alejandra Bruna ${ }^{1}$, Fiorella \\ Campo Verde Arboccó ${ }^{1,2}$, Virginia Pistone Creydt $^{1}$, Constanza Matilde López Fontana ${ }^{1}$ and Rubén \\ Walter Carón*1 \\ ${ }^{1}$ Institute of Medicine and Experimental Biology of Cuyo (IMBECU), Argentina \\ ${ }^{2}$ University of Mendoza, Argentina
}

*Corresponding author: Rubén Walter Carón, Institute of Medicine and Experimental Biology of Cuyo (IMBECU), CCT-Mendoza CONICET, Mendoza, Argentina

ARTICLE INFO

Received: 慧 March 15, 2019

Published: March 21, 2019

Citation: Flavia Eliana S, Corina Verónica S, Leila Ester Z, Flavia Alejandra B, Fiorella Campo Verde A, Virginia Pistone C, Constanza Matilde López F Rubén Walter Ca. High Intake of Maternal Milk Prevents the Development of Mammary Cancer in Pups Maintaining Elevated Ingestion of Saturated Fat. Biomed J Sci \& Tech Res 16(2)-2019. BJSTR. MS.ID.002826.

\section{ABSTRACT}

Environmental factors in early life have a strong implication on the development of diseases in adult life. Nutritional changes during perinatal life can modify the susceptibility to develop breast carcinoma. In this report, we studied the influence of a diet high in saturated fat in the development of breast cancer, in rats that maintained a differential milk intake during their lactation period. We also analysed the possible mechanisms involved in tumor development. We compared mammary carcinogenesis in Sprague-Dawley adult rats, grown-up in litters of 3 (L3) or 8 (L8) pups per mother during lactation to induce a differential consumption of maternal milk. After weaning all pups were fed with a diet high in saturated fat "HF" (40\% of energy from lipids) until adulthood. At 55 days of age, the animals were treated with a single dose of dimethylbenzanthracene to study tumor latency, incidence and progression. Histological and immunohistochemical studies were performed. We observed that animals that maintained high milk intake (L3) had lower mammary cancer incidence than animals that maintained lower milk consumption (L8; P < 0.05) when exposed to a HF diet. Tumor latency and rate of tumor growth did not show variations between the groups. However, the mitotic index $(\mathrm{P}<0.05)$ and the expression of CD1 were significantly lower, in tumors of L3 respect to L8 $(\mathrm{P}<0.01)$. Animals with greater consumption of breast milk develop tumors that proliferate less and tend to have a more pronounced apoptotic process, although when maintaining a high consumption of saturated fat. These results may explain, in part, the lower incidence observed in this group of animals. Our work reflects the importance of lactation during postnatal life in the prevention of breast cancer, despite other nutritional factors that may act as promoters of tumor development throughout life, such as the consumption of saturated fat.

\section{Introduction}

The understanding of the etiology of the human pathologies is a fundamental paradigm to investigate [1,2]. Environmental factors have the potential to alter the development of a young organism, giving rise to the physiology and metabolism of the adult who is thus strongly affected by the signs of early life [3]. Therefore, the health of an adult is shaped to a large extent from the uterus to early childhood. Obesity, diabetes, hypertension, cardiovascular diseases, asthma, allergies and other conditions such as cancer have origins in pre and early postnatal life [4]. Among the environmental factors to which mammals are exposed during early life, nutrition is of great interest; including the diet during pregnancy, the consumption of maternal milk during lactation, as well as the variety and quality 
of the food received after weaning. The development programming from pre-and postnatal nutrition focuses on the epigenetic [5]. Milk is the first postnatal nutritional stimulus of all mammals since the beginning of extrauterine life until the end of the lactation period. It is a complex secretory product that represents a maternal regulator critical of the development of breeding [6].

In humans, breastfeeding provides short and long-term benefits for both the child and the mother [7], including the protection of the child against a variety of acute and chronic pathologies. These benefits are associated both with the amount of breast milk exclusively consumed, and the duration of breastfeeding. There is evidence that milk functions as a transmitter between the maternal genome of lactation and the epigenetic regulation of the genes of the newborn [8]. Breast cancer is one of the most common types of cancer and is the leading cause of cancer mortality in women around the world [9]. Although cancer is influenced by genetic conditions, there are several risk factors such as diet, overweight and sedentary lifestyle that could be determinants in the development of this disease [10]. Dietary components contribute to the etiology of $30-50 \%$ of all breast cancer $[11,12]$. In a preliminary study, we established an in vivo model of differential milk consumption to investigate the specific role of lactation in the risk of developing mammary carcinoma. We demonstrated that animals maintaining more access to maternal milk during postnatal life generate a lower incidence of mammary cancer in the adulthood [13].

Children, adolescents and young adults adopt eating patterns that can be the basis of their eating habits for a large part of their lives [14]. In this sense, there are social factors that favour a greater consumption of fat and its accumulation [15]. The westernized diet is characterized by a high content of proteins, saturated fat, refined grains, sugar, alcohol, salt and fructose syrup derived from corn, with a reduced consumption associated with fruits and vegetables [16]. The association between the consumption of fats and the development of breast cancer arose from the strong correlation observed in studies since 1966 [17]. The use of animal models demonstrated the influence of fat in the development of mammary tumors, independently of energy intake, which suggests a complex interaction between quality and quantity of dietary fat, obesity, inflammation, genetic background and oncogenesis [1822]. Studies in humans on fat consumption and development of breast cancer are inconsistent. This may be related to the difficulty to differentiate the consumption of fat with total caloric intake and obesity; since obesity is identified as a risk factor for breast cancer among postmenopausal women [23-25].

In this report, we studied the influence of a diet high in saturated fat in the development of mammary cancer, in rats that maintained a differential milk intake during their lactation period. We also analysed the possible mechanisms involved in tumor development. Our hypothesis is that a higher consumption of breast milk prevents the development of breast cancer even in animals that ingested a diet high in saturated fats over time. To test this hypothesis, we compared mammary carcinogenesis in adult rats grown-up in litters of 3 or 8 pups per mother during lactation, and that maintained a diet high in saturated fat from its weaning until adulthood. We found significant differences in the tumor incidence of the animals. In addition, we investigate the expression of some proteins related to mitosis in tumor cells.

\section{Materials and Methods}

\section{Postnatal Litter Size Adjustment}

Female Sprague-Dawley rats bred in our laboratory were used. The animals were kept in a light (lights on 6 am to $8 \mathrm{pm}$ ) and temperature $\left(22-24{ }^{\circ} \mathrm{C}\right)$ controlled room. Animal maintenance and handling were performed according to the NIH guide for the Care and Use of Laboratory Animals (NIH publication no. 8623, revised 1991) and the UK requirements for ethics of animal experimentation (Animals Scientific Procedures, Act 1986). All the experimental procedures were approved by the Animal and Ethics Committee (CICUAL) of the School of Medicine of the National University of Cuyo, Mendoza, Argentina. One-day-old female pups $(n=28)$ born on the same day were distributed at random in litters of different sizes: three (L3) or eight (L8) pups per dam, to induce a differential consumption of maternal milk. The size of the litters was chosen in accordance with previous studies [13,26,27]. Body weight of pups was monitored every 3 days.

\section{High Fat Diet}

After weaning (at day 21), all pups were fed with a diet enriched in saturated fat (HF). The diet "HF" has $40 \%$ of energy from lipids and was prepared by adding $12.1 \mathrm{~g}$ of fat extracted from the first bovine juice per $100 \mathrm{~g}$ of standard food (Cargill, Argentina). In both groups tap water was supplied ad libitum until the end of the experiment. Food and beverage consumption, and body weight were monitored weekly, to characterize energy ingestion and weight gain. In this way we obtained two groups of rats' growths with different levels of lactation who maintained a diet high in saturated fats: L3 ( $\mathrm{n}=13)$ and L8 $(\mathrm{n}=15)$.

\section{Food Consumption}

The food was weighed and placed, on top of standard stainlesssteel cages. After $24 \mathrm{~h}$ the amount of remaining food was recorded, including any pellets at the bottom of the cages. Intake was calculated as the weight (in grams) of the foods provided minus those recovered.

\section{Induction of Mammary Tumors}

At the age of 55 days all rats were treated per os with a single dose (15mg / rat) of 7,12-dimethylbenzanthracene (DMBA, Sigma-Aldrich, USA) by an intragastric probe, $3 \mathrm{~h}$ after food and water deprivation to ensure a complete absorption of the drug. DMBA has been extensively used at that dose to study mammary carcinogenesis [28-30]. The animals were palpated twice a week. 
Latency, incidence and progression of tumors were determined in all groups.

\section{Latency, Incidence and Progression of Tumors}

The latency was considered as the time between DMBA administration and the appearance of the first palpable tumor. Incidence was calculated as the percentage of rats that had tumors within the study period with respect to the total number of rats per group. We used a caliper measure the major (DM) and minor $(\mathrm{dm})$ diameters of the tumors twice a week and calculated the tumor volume (TV) as TV $=\mathrm{dm}^{2} \times \mathrm{DM} / 2$. Tumor progression was assessed estimating tumor growth rate (GR) as GR = TV / (day of sacrifice-day of appearance of first tumor).

\section{Sample Collection}

All the animals were decapitated between 10 am and 12 noon on the day of diestrus when the tumors reached a volume $\bigotimes 1000$ $\mathrm{mm}^{3}$ or at the end of the experiment on day 250 if they did not develop any mammary tumor $[13,31]$. When more than one tumor developed in a single rat, the volume limit of $1000 \mathrm{~mm} 3$ was considered for the first tumor reaching that value. Immediately after decapitation, intraabdominal fat was removed, weighed and expressed as a percentage of total body weight. A piece of each tumor was removed for histopathological and immunohistochemical (IHC) analysis.

\section{Immunohistochemistry}

Serial sections (3-5 $\mu \mathrm{m})$ were mounted onto 3-aminopropyltriethoxysilane (Sigma-Aldrich, Argentina) -coated slides for subsequent IHC analysis. An antigen retrieval protocol using heat to unmask the antigens was used (30 min in citrate buffer, $0.01 \mathrm{M}, \mathrm{pH}$ 6.0). Tissue sections of 9-14 tumors / group were incubated overnight at $4{ }^{\circ} \mathrm{C}$ in humidity chambers with the primary anti-proliferating cell nuclear antigen (PCNA, Dako Cytomation, Denmark), anti-Ki67 (Abcam, USA) and anti-cyclin D1 (CD1, Abcam, USA). A commercial kit to detect mouse antibody was used (Dako EnVision system, horseradish peroxidase, diaminobenzidine, Dako, USA).
Slides were lightly counterstained with hematoxylin to reveal the nuclei, examined and photographed. The percentage of positive nuclei was obtained based on an average of 700 cells counted per sample, at $400 \times$ magnification. We used a scoring system reported previously [32]. Briefly, we used an intensity score $0=$ no staining, $1=$ nuclear staining of $<10 \%$ of tumor cells, 2 = staining between 11 and $33 \%$ of tumor cells, 3 = staining between 34 and $65 \%$ of tumor cells, $4=$ staining of $>66 \%$ of tumor cells. These scores were obtained by two independent observers blinded on the experimental group, and a few conflicting scores were resolved by consensus.

\section{Statistical Analysis}

Values are given as means \pm S.E.M. of 13-15 animals per group. All statistical analyses were performed using GraphPad Prism 5.01 software (GraphPad Software Inc., USA). The data were analyzed by the student $T$ test for independent samples or Mann Whitney U test according to the criteria of normality of the variables established with the Kolmogorov-Smirnov normality test. The means of the body weight (BW) gain of the litters in function of time were compared using two-way analysis of variance (ANOVA II). The incidence percentages were analysed by Fisher's test. Differences between means were considered significant at the $\mathrm{P}<0.05$ level.

\section{Results}

\section{Body Weight}

The growth curve of the animals with differential lactation shows a significant increase in weight $(\mathrm{P}<0.001)$ in $\mathrm{L} 3$ with respect to L8 from day 11 of life and until the end of lactation (Figure 1a). These results agree with those previously published [13]. This difference in weight gain is given exclusively by the consumption of breast milk. Figure 1b shows a greater increase in BW $(\mathrm{P}<0.05)$ in L3 with respect to L8 from weaning and up to week 15 of life ( $\mathrm{P}<0.05)$, supporting that the increase in BW produced by a high milk consumption during lactation is maintained even during the post-weaning period. The consumption of a HF diet evens up the BW of both experimental groups after the 15th week of life and until the end of the experiment (Figure 1).
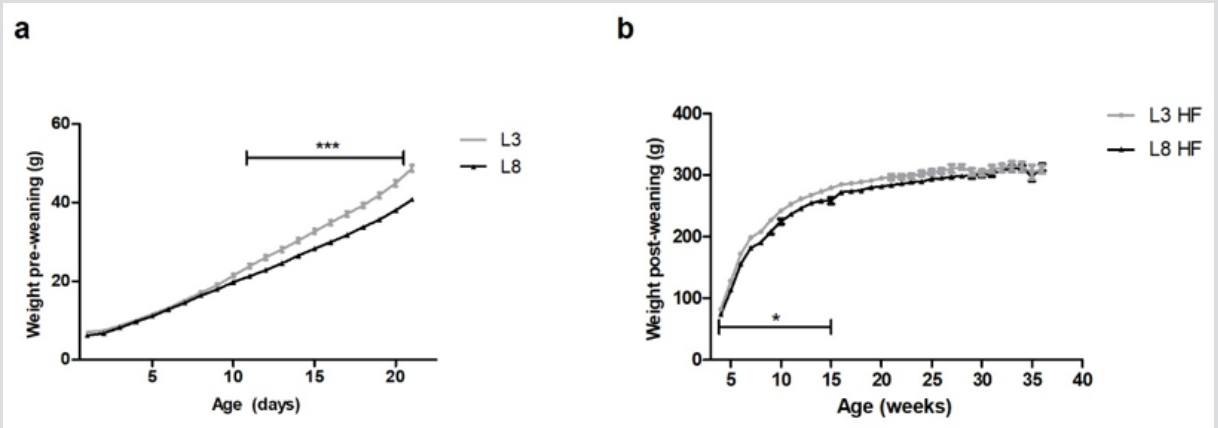

Figure 1: Pre and post-weaning BW of rats with differential milk consumption, that maintained a HF diet.

(a) The variations in weight between the two groups can be observed from day 11 of life onward $\left({ }^{* * *} \mathrm{P}<0.001\right)$ during the lactation period.

(b) The L3 pups have a greater weight with respect to the L8 pups during weeks 4 to 15 of life $\left({ }^{*} \mathrm{P}<0.05\right)$. As of week, 15 of age, no differences were observed in the weights between L3 and L8. The values represent the mean \pm SEM of 13-15 animals per group. The comparisons were made by ANOVAII. 


\section{Food Consumption}

The measurement of the intake of HF diet during the postweaning period (week 4 of life and until the end of the experiment) showed no difference between differentially breastfed animals (L3 and L8) (Figure 2).

\section{Intra-Abdominal Fat Mass}

The intra-abdominal fat mass was extracted at the time of sacrifice of the animals and was expressed as a percentage of BW. The percentage of abdominal fat was similar among the experimental groups studied (Figure 3).

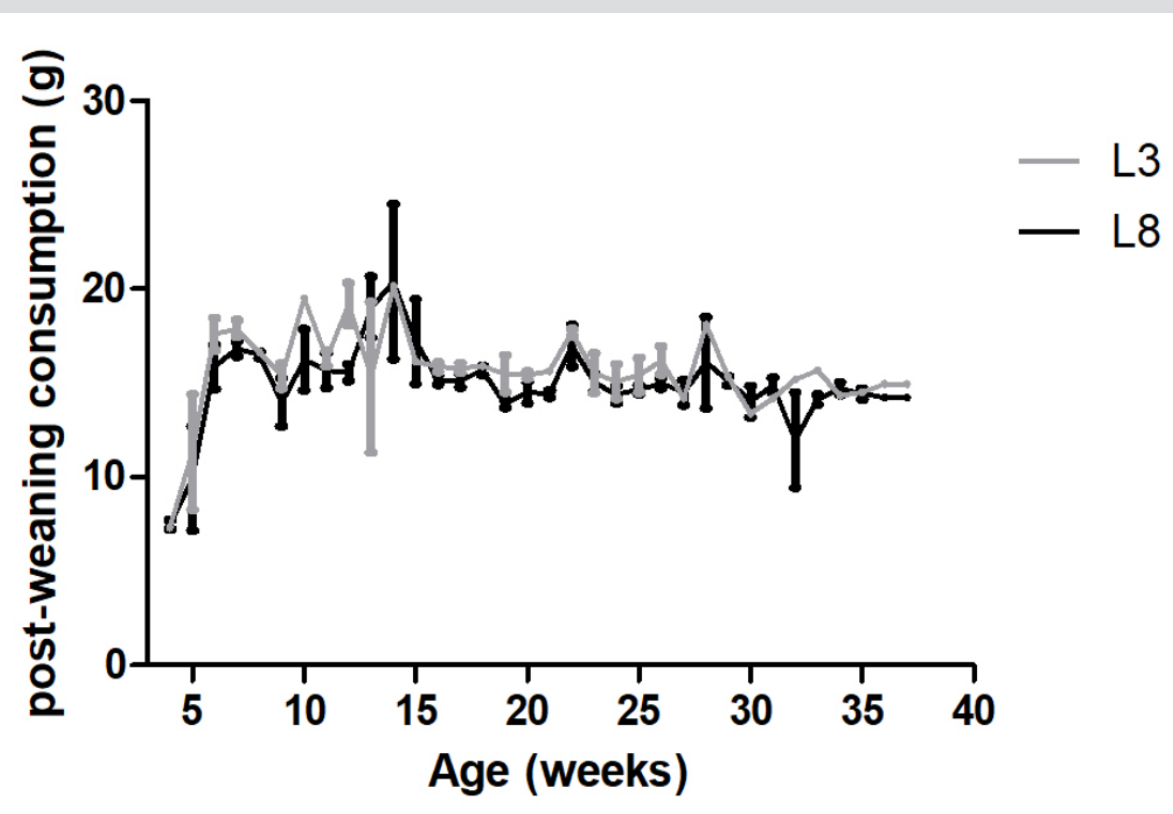

Figure 2: The consumption of HF diet is similar in animals with different ingestion of maternal milk. The values represent the mean \pm SEM of 13-15 animals per group. The comparisons were made by ANOVA II.

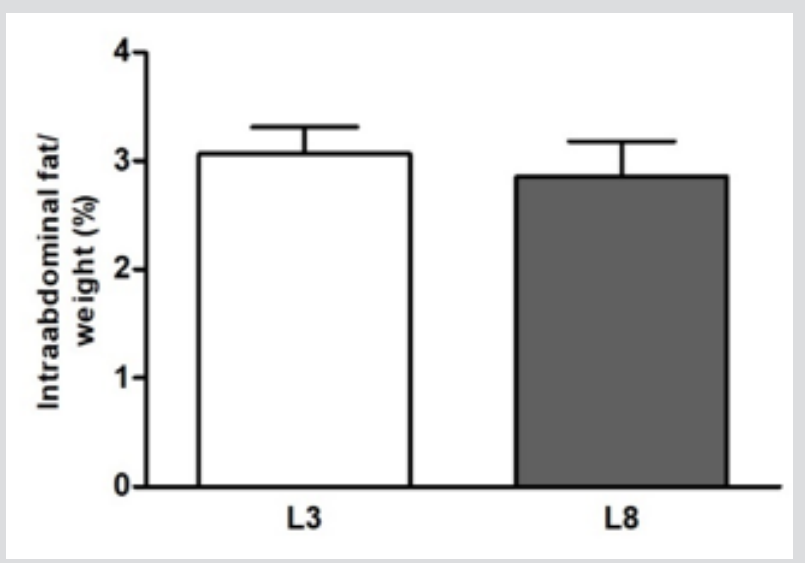

Figure 3: The Percentage of intra-abdominal fat mass is similar in animals with HF diet and different ingestion of maternal milk. No differences were found in the percentage of intraabdominal fat mass between L3 and L8. The values represent the weight of the fatty tissue expressed as a percentage of the BW of 13-15 animals / group. The comparison was made using the Student's T test for independent samples.

\section{Mammary Carcinogenesis}

We proceeded to analyse the percentage of rats that generated tumors within the studied period. The results obtained show that animals that maintained high milk intake (L3) had lower mammary cancer incidence than animals that maintained lower milk consumption (L8; $\mathrm{P}<0.05$, Figure 4a) when exposed to a HF diet. On the other hand, tumor latency did not show variations between L3 and L8 compared to the constant influence of a HF diet (Figure 4b). We also analysed the rate of tumor growth which was expressed in $\mathrm{mm}^{3}$ / day. Differential maternal milk intake did not significantly modify the growth rate of the tumors between the two groups (Figure 4c). That animals that maintained high milk intake (L3) had lower mammary cancer incidence than animals that maintained lower milk consumption (L8; $\mathrm{P}<0.05$, Figure 4a) when exposed to a HF diet. 

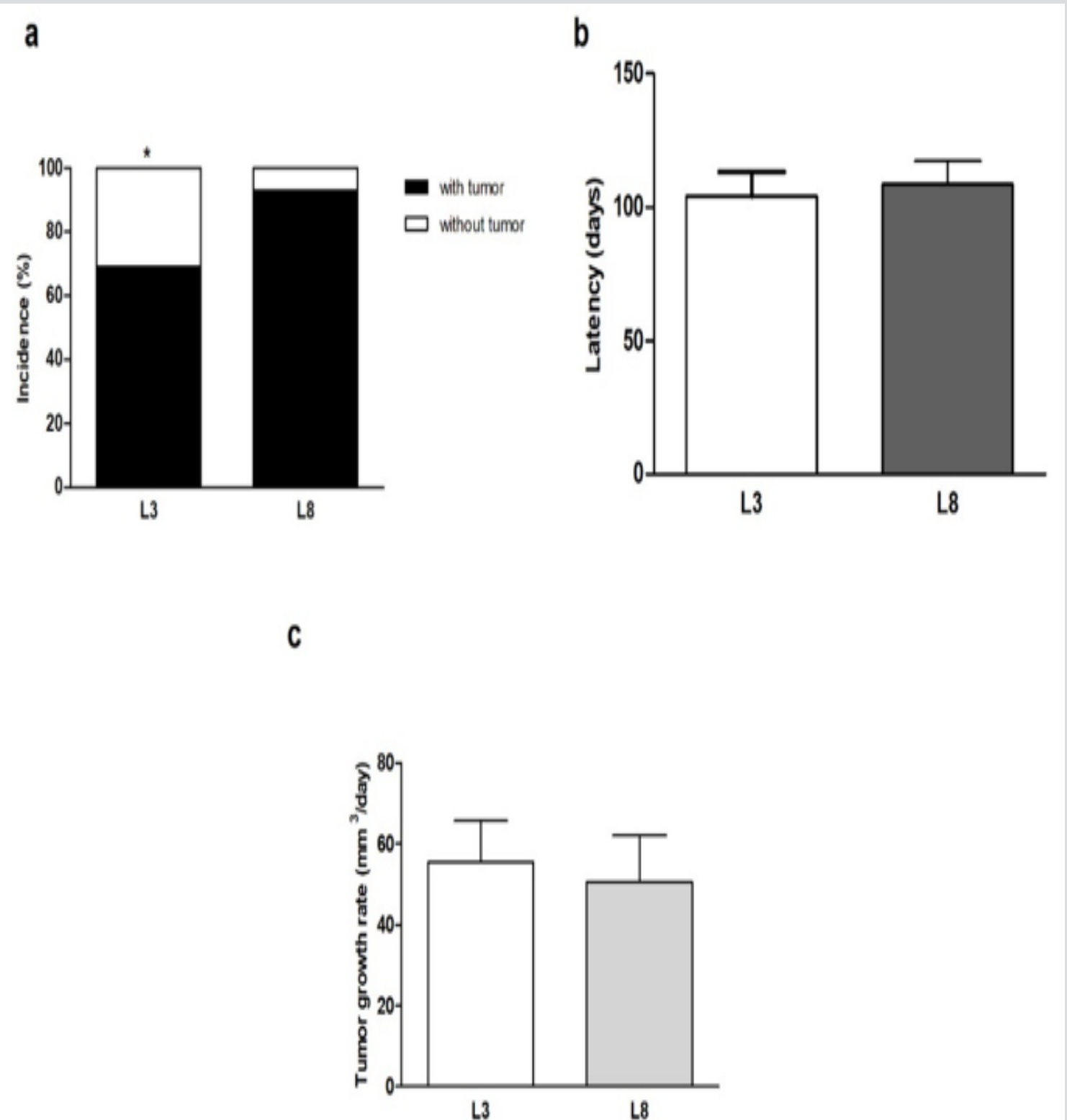

Figure 4: High milk intake decreases tumor incidence in a diet high in saturated fat.

(a) The incidence of breast cancer in animals with HF diet is lower in L3 than in L8 (* $\mathrm{P}<0.05)$.

(b) No differences were observed in mammary tumor latency between L3 and L8.

(c) The rate of tumor growth was similar between L3 and L8. Values represent mean \pm S.E.M. of 13-15 animals / group.

Incidence was expressed as percentages and compared by Fisher's test. Comparisons in latency was performed by the Student $\mathrm{T}$ test for independent samples. Tumor growth was compared by the nonparametric Mann-Whitney test. L3, litter of three pups per mother; L8, litter of eight pups per mother.

\section{Tumor Proliferation}

In order to evaluate the effect of fat consumption on tumor proliferation in animals with differential lactation, we performed a microscopic analysis of mitosis and apoptosis. The mitotic index was significantly lower $(\mathrm{P}<0.05$, Figure $5 \mathrm{a})$ in $\mathrm{L} 3$ respect to $\mathrm{L} 8$. The apoptotic index (Figure 5b) did not show significant differences, however, showed a tendency to be increased in tumors of L3 versus L8. To investigate whether the balance between mitosis and apoptosis influences tumor development, we calculated the mitotic / apoptotic ratio. This parameter tended to be increased in group L8 (Figure 5c). We additionally evaluated mitosis by IHC of PCNA, Ki67 and CD1 in the tumors. No changes were observed in the expression of PCNA (Figure 6a) and Ki67 (Figure 6b) between the groups studied. The expression of CD1 was increased in L8 with respect to L3 $(\mathrm{P}<0.01$, Figure $6 \mathrm{c})$. 
a

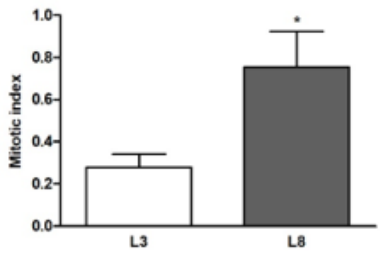

b

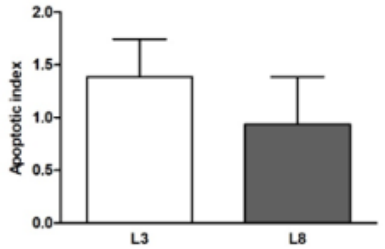

C

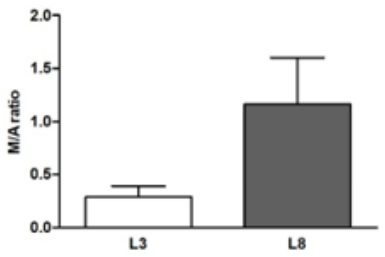

Figure 5: High milk intake decreases mitosis and tends to increase apoptosis in the tumors of animals that maintained a HF diet.

(a) The mitotic index was higher in tumors of L8 compared to L3 $(\mathrm{P}<0.05)$.

(b) The apoptotic index tended to be increased in L3 versus L8 tumors.

(c) The mitotic / apoptotic ratio tended to be higher in L8 when compared to L3.

Values are means \pm S.E.M. of of 8-10 fields of each preparation from 9 to 14 animals/group. Comparisons were performed by the non-parametric U Mann Whitney test. L3, litter of three pups per mother; L8, litter of eight pups per mother.

\section{a}

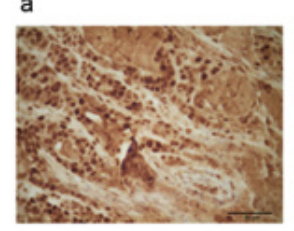

b
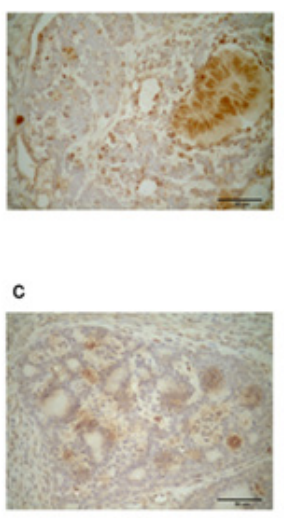
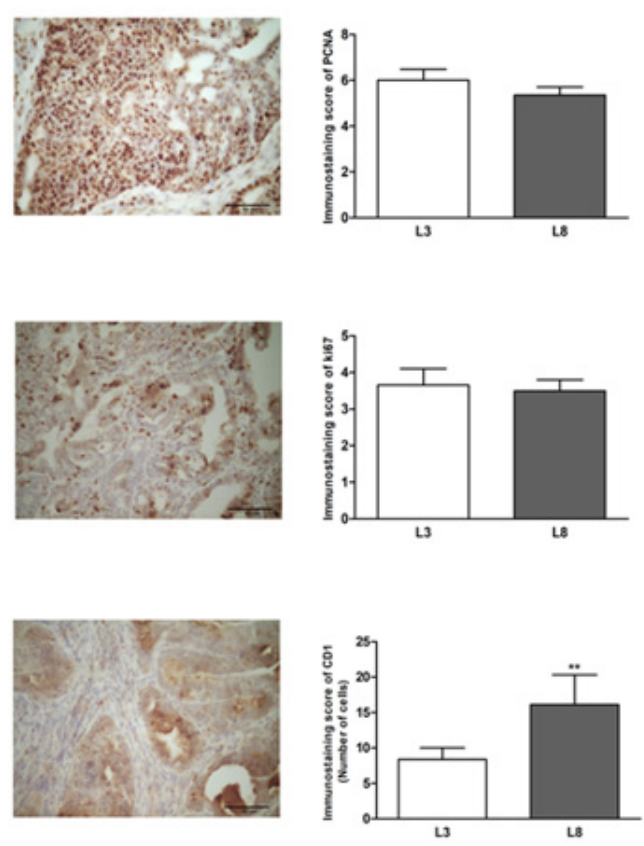

Figure 6: High milk intake reduces expression of CD1 in animals that maintained a diet high in saturated fat. (a-c) Representative microphotographs $(400 \times)$ of tumors immunoassayed to reveal PCNA, Ki67 and CD1. Histograms show mean \pm SEM of 8-10 fields of each preparation from 9 to 14 animals / group.

(a) PCNA-immunostaining score. The expression of PCNA determined by IHC was similar in the two groups.

(b) Ki67-immunostaining score. No differences were observed in Ki67 expression between L3 and L8.

(c) The expression of CD1 was increased in L8 with respect to L3 (** $\mathrm{P}<0.01$ ).

The results were expressed as a percentage of CD1 positive cells. Comparisons were performed by the nonparametric U Mann Whitney test for PCNA and Ki67 and the Student T test for independent samples for CD1. 


\section{Discussion}

Environmental factors in early life have a strong implication on the development of diseases in adult life. Epidemiological studies in humans and research in animal models have shown that nutritional changes during early life can modify the susceptibility to develop breast carcinoma [3]. The mechanisms by which early nutrition can induce stable physiological or metabolic changes involve epigenetic processes capable of altering gene expression $[3,33]$. In this sense, feeding during childhood and preadolescence can affect growth and development parameters of adolescents, such as the age at first menarche, and the age and amplitude of the peak of growth. Each of these parameters act independently as risk factors associated with the development of breast cancer [34]. The peak height growth velocity has been positively associated with breast cancer risk [35]. Whereas, breast cancer risk increased for every year at menarche [36]. While the evidence from the studies regarding the association of total and subtypes of fat intake with breast cancer risk are inconsistent, several studies have shown that saturated fat intake is associated with an increased risk of breast cancer.

The Women's Health Initiative study found a tendency to reduce the risk of breast cancer in post-menopausal women who maintained a low-fat diet [37]. Accordingly, diets high in saturated fat were associated with an increased risk of developing estrogen receptor positive breast cancer [38]. Several case-control studies have found a positive association between the consumption of saturated fats and the risk of developing breast cancer [39-42]. On the other hand, a meta-analysis of 11 cohort studies found no association between saturated fat intake and breast cancer [43]. In a preliminary study we demonstrated that animals maintaining more access to maternal milk during postnatal life showed a lower incidence of mammary cancer in the adulthood [13]. This work allowed us to identify the effect over time of variations in postnatal maternal milk consumption, with a controlled animal model. We know that, extrapolated to humans, the benefits of breast milk consumption can be influenced by a number of other nutritional factors after weaning. To have a more complete knowledge on the subject, we wanted to study the influence of a diet with a high content of saturated fat (simulating a Western diet) from the moment of weaning in those animals that had consumed different amounts of breast milk during their lactation.

This allowed us to get closer to the complex food interaction that the human being experiences in critical periods of its development. We observed that the greater consumption of breast milk during postnatal life prevents the development of breast cancer, even when a high intake of saturated fat is maintained over time. This suggests that a greater consumption of breast milk can activate epigenetic, physiological and / or metabolic changes that decrease the susceptibility of the mammary gland to the cancerous development even in the presence of promoter stimulus such as the high-fat diet. The development and progression of cancer is strongly influenced by the proliferation process. This is manifested by altered expression and / or activity of cell cycle related proteins [44]. In the present study, we analysed cell proliferation through the mitosis count and the detection by immunohistochemical staining of cell cycle proteins [45]. We observed that L8 animals had more proliferative tumors compared to L3, evidenced by the greater number of mitotic bodies and the greater expression of CD1 observed in the tumor sections. This suggests that animals with greater consumption of breast milk develop tumors that proliferate less and tend to have a more pronounced apoptotic process, although when maintaining a high consumption of saturated fat.

These results may explain, in part, the lower incidence observed in this group of animals. Although the animals with the highest consumption of breast milk developed less proliferative tumors, no differences were observed in the tumor growth rate. Our work reflects the importance of lactation during postnatal life in the prevention of breast cancer, despite other nutritional factors that may act as promoters of tumor development throughout life, such as the consumption of saturated fat. The animal model used allowed us to study in depth the influence of different consumption of breast milk, and the influence of the ingestion of a high-fat diet simulating a western style of feeding. It is necessary to delve into the possible mechanisms involved by the components of breast milk on the development of mammary epithelium and carcinogenesis. It is of great interest to detect altered epigenetic marks in early life that can provide biomarkers to detect individuals at higher risk of disease.

\section{Acknowledgment}

The authors are deeply indebted to Vet. Paula Ginevro and $\mathrm{Mr}$ Juan Rosales for their excellent technical assistance.

\section{Financial Support}

This work was supported by a grant of Instituto Nacional del Cáncer, identified as number B11 according to resolution of the Ministry of Health of Argentina number 493/14.

\section{Conflicts of Interest}

The authors declare that there is no conflict of interest that could be perceived as prejudicing the impartiality of the research reported.

\section{References}

1. Barker DJP (1998) In utero programming of chronic disease. Clin Sci 95(2): 115-128.

2. Gluckman PD, Hanson MA (2004) Maternal constraint of fetal growth and its consequences. Seminars in Fetal and Neonatal Medicine 9(5): 419-425.

3. Lillycrop KA, Burdge GC (2014) Breast Cancer and the Importance of Early Life Nutrition. Advances in Nutrition and Cancer. Advances in Nutrition and Cancer, pp. 269-285.

4. Gray K, Landrigan PJ, Suk W, Thompson C, Grandjean P, et al. (2015) Developmental Origins of Health and Disease: Integrating Environmental Influences. Endocrinology 156(10): 3416-3421.

5. Vickers MH (2014) Early life nutrition, epigenetics and programming of later life disease. Nutrients 6(6): 2165-2178. 
6. Power ML, Schulkin J (2013) Maternal regulation of offspring development in mammals is an ancient adaptation tied to lactation. Appl Transl Genomics 2: 55-63.

7. León Cava N, Lutter C, Ross J, Martin L (2002) Cuantificación de los Beneficios de la Lactancia Materna: Reseña de la Evidencia. Us11, pp. 1-186.

8. Melnik BC, John SM, Schmitz G (2013) Milk is not just food but most likely a genetic transfection system activating mTORC1 signaling for postnatal growth. Nutr J 12(1): 103.

9. Siegel RL, Miller KD, Jemal A (2019) Cancer statistics, 2019. CA Cancer J Clin 69(1): 7-34.

10. Kerr J, Anderson C, Lippman SM (2017) Physical activity, sedentary behaviour, diet, and cancer: an update and emerging new evidence. Lancet Oncol 18(8): 457-471.

11. Hilakivi Clarke L (2007) Nutritional Modulation of Terminal End Buds: Its Relevance to Breast Cancer Prevention. Current Cancer Drug Targets 7(5): 465-474.

12. Heitz AE, Baumgartner RN, Baumgartner KB, Boone SD (2018) Healthy lifestyle impact on breast cancer-specific and all-cause mortality. Breast Cancer Res Treat 167(1): 171-181.

13. Santiano FE, Zyla LE, Campo Verde Arboccó F, Sasso CV, Bruna FA, et al. (2019) High maternal milk intake in the postnatal life reduces the incidence of breast cancer during adulthood in rats. J Dev Orig Health Dis 10: 1-9.

14. Bull NL (1992) Dietary habits, food consumption, and nutrient intake during adolescence. J Adolesc Heal 13(5): 384-348.

15. Siddarth D (2013) Risk factors for obesity in children and adults. J Investig Med 61(6): 1039-1042.

16. Statovci D, Aguilera M, MacSharry J, Melgar S (2017) The Impact of Western Diet and Nutrients on the Microbiota and Immune Response at Mucosal Interfaces. Front Immunol 8: 838

17. Lea AJ (1996) Dietary factors associated with death-rates from certain neoplasms in man. Lancet 2(7458): 332-333.

18. Carroll KK, Khor HT (1975) Dietary fat in relation to tumorigenesis. Prog Biochem Pharmacol 10: 308-353.

19. Freedman LS, Clifford C, Messina M (1990) Analysis of Dietary Fat, Calories, Body Weight, and the Development of Mammary Tumors in Rats and Mice: A Review. Cancer Res 50(18): 5710-5719.

20. Thaiss CA, Zmora N, Levy M, Elinav E (2016) The microbiome and innate immunity 535(7610): 65-74

21. Beyaz S, Mana MD, Roper J, Kedrin D, Saadatpour A, et al. (2016) Highfat diet enhances stemness and tumorigenicity of intestinal progenitors. Nature 531(7592): 53-58.

22. Di Daniele N, Noce A, Vidiri MF, Moriconi E, Marrone G, et al. (2017) Impact of Mediterranean diet on metabolic syndrome, cancer and longevity. Oncotarget 8(5): 8947-8979.

23. Cummings JH, Bingham SA (1988) Diet and the prevention of cancer. BMJ 317(7173): 1636-1640.

24. Freedman LS, Potischman N, Kipnis V, Midthune D, Schatzkin A, et al. (2006) A comparison of two dietary instruments for evaluating the fatbreast cancer relationship. Int J Epidemiol 35(4): 1011-1021.

25. Thiébaut ACM, Kipnis V, Chang SC, Subar AF, Thompson FE, et al. (2007) Dietary fat and postmenopausal invasive breast cancer in the National Institutes of Health-AARP Diet and Health Study cohort. J Natl Cancer Inst 99(6): 451-462.

26. Velkoska E, Cole TJ, Morris MJ (2005) Early dietary intervention: longterm effects on blood pressure, brain neuropeptide $\mathrm{Y}$, and adiposity markers. Am J Physiol Metab 288(6): 1236-1243.
27. Chen H, Simar D, Lambert K, Mercier J, Morris MJ (2008) Maternal and Postnatal Overnutrition Differentially Impact Appetite Regulators and Fuel Metabolism. Endocrinology 149(11): 5348-5356.

28. Barros ACSD, Muranaka ENK, Mori LJ, Pelizon CHT, Iriya K, et al. (2004) Induction of experimental mammary carcinogenesis in rats with 7,12-dimethylbenz(a)anthracene. Revista do Hospital das Clínicas 59(5): 257-261.

29. Jahn GA, Diolez Bojda F, Belair L, Kerdelhué B, Kelly PA, et al. (1991) Effect of DMBA on the expression of prolactin receptors and IGF1 genes in rat mammary gland. Biomed Pharmacother 45(1): 15-22.

30. Russo J, Russo IH (1996) Experimentally induced mammary tumors in rats. Breast Cancer Res Treat 39(1): 7-20.

31. Sasso CV, Santiano FE, Lopez-Fontana CM, Pistone Creydt V, Ezquer ME et al. (2014) Effects of parity and serum prolactin levels on the incidence and regression of DMBA-induced tumors in OFA hr/hr rats. Biomed Res Int 2014: 210424

32. Gago FE, Tello OM, Diblasi AM, Ciocca DR (1998) Integration of estrogen and progesterone receptors with pathological and molecular prognostic factors in breast cancer patients. J Steroid Biochem Mol Biol 67(5-6): 431-437.

33. Melnik BC, Schmitz G (2017) Milk's Role as an Epigenetic Regulator in Health and Disease. Dis 5(1): 12.

34. Bertrand KA, Gerlovin H, Bethea TN, Palmer JR (2017) Pubertal growth and adult height in relation to breast cancer risk in African American women. Int J Cancer 141(12): 2462-2470.

35. Colditz GA, Bohlke K, Berkey CS (2014) Breast cancer risk accumulation starts early: prevention must also. Breast Cancer Res Treat 145(3): 567579.

36. Collaborative Group on Hormonal Factors in Breast Cancer (2012) Menarche, menopause, and breast cancer risk: individual participant meta-analysis, including 118964 women with breast cancer from 117 epidemiological studies. Lancet Oncol 13(11): 1141-1151.

37. Prentice RL, Caan B, Chlebowski RT, Patterson R, Kuller LH, et al. (2006) Low-fat dietary pattern and risk of invasive breast cancer: The Women's Health Initiative Randomized Controlled Dietary Modification Trial. JAMA 295(6): 629-642.

38. Sieri S, Chiodini P, Agnoli C, Pala V, Berrino F, et al. (2014) Dietary Fat Intake and Development of Specific Breast Cancer Subtypes. JNCI J Natl Cancer Inst 106(5).

39. Boyd NF, Stone J, Vogt KN, Connelly BS, et al. (2003) Dietary fat and breast cancer risk revisited: a meta-analysis of the published literature. Br J Cancer 89(9): 1672-1685.

40. Hunter DJ, Spiegelman D, Adami HO, Beeson L, Van Den Brandt PA, et al. (1996) Cohort studies of fat intake and the risk of breast cancer--a pooled analysis. N Engl J Med 334(6): 356-361.

41. Ronco A, De Stefani E, Mendilaharsu M, Deneo Pellegrini H (1996) Meat, fat and risk of breast cancer: a case-control study from Uruguay. Int J cancer 65(3): 328-331

42. Wakai K, Dillon DS, Ohno Y, Prihartono J, Budiningsih S, et al. (2000) Fat intake and breast cancer risk in an area where fat intake is low: a casecontrol study in Indonesia. Int J Epidemiol 29(1): 20-28.

43. Alexander DD, Morimoto LM, Mink PJ, Lowe KA (2010) Summary and meta-analysis of prospective studies of animal fat intake and breast cancer. Nutr Res Rev 23(1): 169-179.

44. Feitelson MA, Arzumanyan A, Kulathinal RJ, Blain SW, Holcombe RF et al. (2015) Sustained proliferation in cancer: Mechanisms and novel therapeutic targets. Semin Cancer Biol 35(S): 25-54.

45. Elias JM (1997) Cell proliferation indexes: a biomarker in solid tumors. Biotech Histochem 72(2): 78-85. 


\section{ISSN: 2574-1241}

DOI: 10.26717/BJSTR.2019.16.002826

Rubén Walter Carón. Biomed J Sci \& Tech Res

(c) This work is licensed under Creative

Submission Link: https://biomedres.us/submit-manuscript.php

$\begin{array}{ll}\text { BIOMEDICAL } & \text { Assets of Publishing with us } \\ \text { RESEARCHES } & \text { Global archiving of articles } \\ \text { - Immediate, unrestricted online access }\end{array}$

